\title{
The WEAVE prime focus Correction: from design to integration
}

\section{Albert Tomàs, Manuel Canchado, Joan Manel Casalta, Francesc Dalmases, Oscar Maroto, et al.}

Albert Tomàs, Manuel Canchado, Joan Manel Casalta, Francesc Dalmases, Oscar Maroto, Carlos Martín-Nuño, Antonio Romero, J. Alfonso L. Aguerri, José Miguel Herreros, José Miguel Delgado, José Alonso Burgal, Don Carlos Abrams, Kevin Dee, Emilie Lhomé, Gavin Dalton, Kevin Middleton, Piercarlo Bonifacio, Scott C. Trager, Antonella Vallenari, Esperanza Carrasco, "The WEAVE prime focus Correction: from design to integration," Proc. SPIE 10706, Advances in Optical and Mechanical Technologies for Telescopes and Instrumentation III, 1070606 (10 July 2018); doi: $10.1117 / 12.2313718$

SPIE Event: SPIE Astronomical Telescopes + Instrumentation, 2018, Austin, Texas, United States 


\title{
The WEAVE Prime Focus Corrector: from Design to Integration
}

\author{
Albert Tomás ${ }^{\mathrm{a}}$, Manuel Canchado ${ }^{\mathrm{a}}$, Joan Manel Casalta ${ }^{\mathrm{a}}$, Francesc Dalmases ${ }^{\mathrm{a}}$, Oscar Maroto ${ }^{\mathrm{a}}$, Carlos \\ Martín-Nuño $^{\mathrm{a}}$, Antonio Romero ${ }^{\mathrm{a}}$, J. Alfonso L. Aguerri ${ }^{\mathrm{b}}$, José Miguel Herreros ${ }^{\mathrm{b}}$, José Miguel \\ Delgado $^{b}$, José Alonso Burgal ${ }^{b}$, Don Carlos Abrams ${ }^{\mathrm{c}}$, Kevin Dee ${ }^{\mathrm{c}}$, Emilie Lhoméc, Gavin Dalton ${ }^{\mathrm{d}, \mathrm{e}}$, \\ Kevin Middleton ${ }^{\mathrm{e}}$, Piercarlo Bonifacio ${ }^{\mathrm{f}}$, Scott C. Trager ${ }^{\mathrm{g}}$, Antonella Vallenari ${ }^{\mathrm{h}}$, Esperanza Carrasco ${ }^{\mathrm{i}}$ \\ a SENER Ingeniería y Sistemas, C/ Creu Casas i Sicart, 86-88, Parc de 1'Alba 08290 Cerdanyola del \\ Vallès, Barcelona, Spain; \\ ${ }^{\mathrm{b}}$ Instituto de Astrofísica de Canarias (IAC), Vía Lactea S/N, Tenerife, Spain; \\ ${ }^{\mathrm{c}}$ Isaac Newton Group of Telescopes, Apartado de Correos 321, 38700 Santa Cruz de la Palma, \\ Canary Islands, Spain; \\ ${ }^{d}$ Dept. of Physics, University of Oxford, Keble Road, Oxford, OX1 3RH, UK; \\ ${ }^{\mathrm{e}}$ RALSpace, STFC Rutherford Appleton Laboratory, OX11 0QX, UK; \\ ${ }^{f}$ GEPI, Observatoire de Paris, 61 avenue de l'Observatoire, 75014 Paris, France; \\ ${ }^{g}$ Kapteyn Institut, Rijksuniversiteit Groningen, Postbus 800, NL-9700 AV Groningen, Netherlands; \\ ${ }^{\mathrm{h}}$ INAF, Observatorio Astronomico di Padova, Vicolo Observatorio 5, 35122 - Padova, Italy; \\ i INAOE, Luis Enrique Erro 1, Tonantzintla, Puebla, Mexico.
}

\begin{abstract}
WEAVE is a new wide-field multi-object spectroscopy (MOS) facility proposed for the prime focus of the $4.2 \mathrm{~m}$ William Herschel Telescope (WHT), situated on the island of La Palma, Canary Islands, Spain. To allow for the compensation of the effects of temperature-induced and gravity-induced image degradation, the WEAVE prime focus assembly will be translated along the telescope optical axis. The assembly comprises the prime focus corrector (PFC), a central mount for the corrector known as FTS ${ }^{[1]}$, an instrument rotator and a twin-focal-plane fibre positioner. SENER, that manufactured and delivered the FTS, is also responsible for the final design, manufacturing, integration, alignment and testing of the PFC and its ancillary equipment. This manuscript describes the final design of the PFC along with the analyses and simulations performed and presents the procedures for the integration and alignment of the lenses in the corrector.
\end{abstract}

Keywords: PFC, ADC, corrector, WEAVE, WHT, AIT

\section{INTRODUCTION}

The WEAVE ${ }^{[2]}$ facility comprises a new 2-degree field-of-view Prime Focus Corrector (PFC) with a 1000-multiplex fibre positioner, a small number of individually deployable integral field units, and a large single integral field unit (IFU). The IFUs and the MOS fibres can be used to feed a dual-beam spectrograph that will provide full coverage of the majority of the visible spectrum in a single exposure at a spectral resolution of $\sim 5000$ or modest wavelength coverage in both arms at a resolution $\sim 20000$. The instrument will provide spectroscopic sampling of the fainter end of the Gaia astrometric catalogue, chemical labelling of stars to $\mathrm{V} \sim 17$, and dedicated follow-up of substantial numbers of sources from the medium deep LOFAR surveys.

The PFC is an opto-mechanical sub-system of $1700 \mathrm{~kg}$ consisting of a structural housing supporting six lens assemblies. It integrates an Atmospheric Dispersion Corrector (ADC) which consists of two doublets (four lenses in total), tilted on assembly with respect to the optical axis (Figure 1). These doublets are mounted in a driving mechanism that provides rotation around the optical axis. Both ADC's rotate in opposite direction compensating for the atmospheric dispersion caused by the change of telescope elevation angle. The doublets driving mechanism offers accuracies up to 0.05 degree. 
To maintain the required image quality, the lenses are aligned with tolerances of less than $50 \mu \mathrm{m}$. The main functionalities of the PFC are:

- Provide stable support for the optical lenses for the applicable loads (gravitational and thermal).

- Provide low stressed support for the optical lenses $(<3.4 \mathrm{MPa})$ to prevent optical aberrations.

- Provide the driving system for both doublets with the required accuracy, repeatability and resolution.

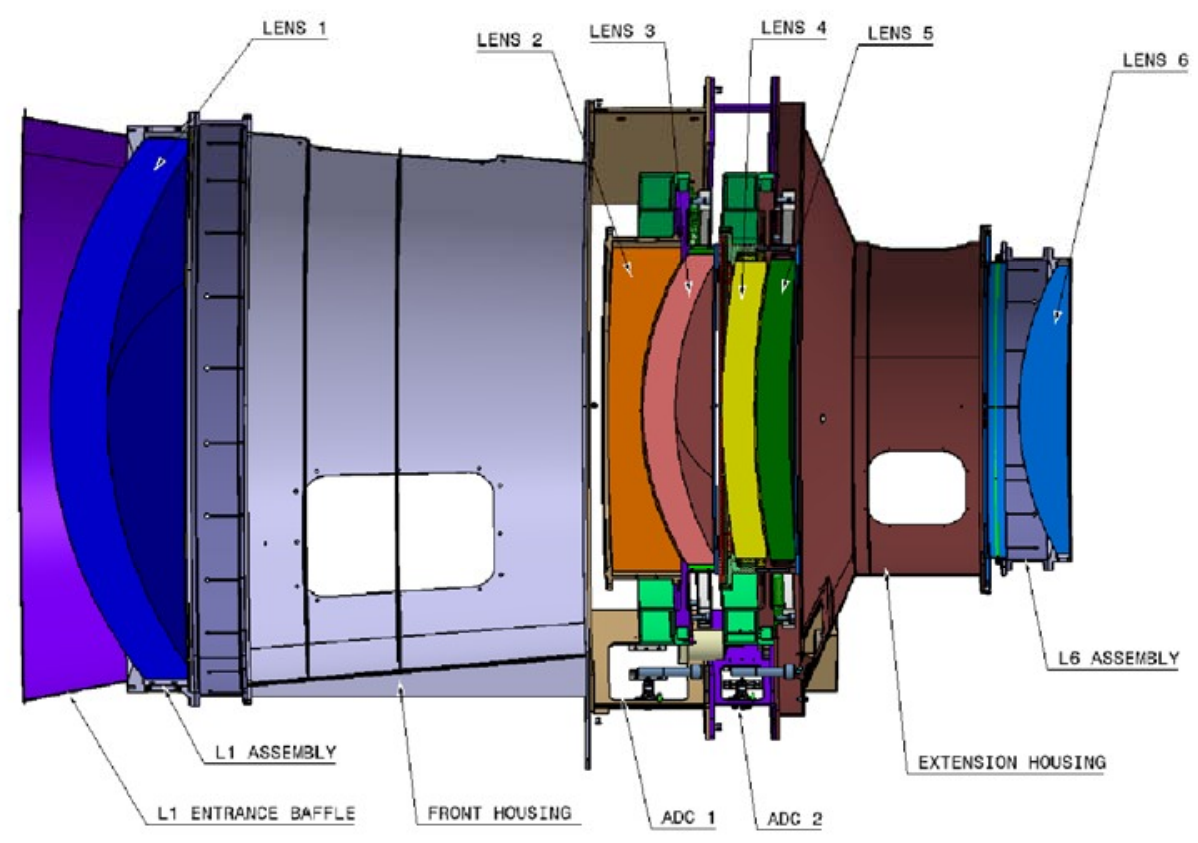

Figure 1. Schematic cut through the PFC

\subsection{Housing and Lens Cell design}

\section{PFC DESIGN}

The PFC structure is made by a set of parts that holds the lenses. Each lens is mounted into a lens cell that provides interface to the PFC housings. L1 cell and L6 cell at both extremes of the corrector are connected to the four central housings pieces. Inside the housing there are the two doublets (ADC's). The ADC's are connected to the central housings through the support outer cylinders and the bearings. Besides the main structure there are the internal and external baffles to prevent the stray light.

The lenses are fixed to their support structures (cells) with discrete silicone pads (RTV560) in axial and radial direction (Figure 2 with L1 example) to provide a thermal support. Axial pads hold the lens to the cell and radial pads fix the position of the lens relative to the cell. Radial pads are installed in the assembly through inserts. This elastic solution keeps the lens stress below 3.4MPa while minimizing the gravitational displacement for the different telescope elevation angles. 


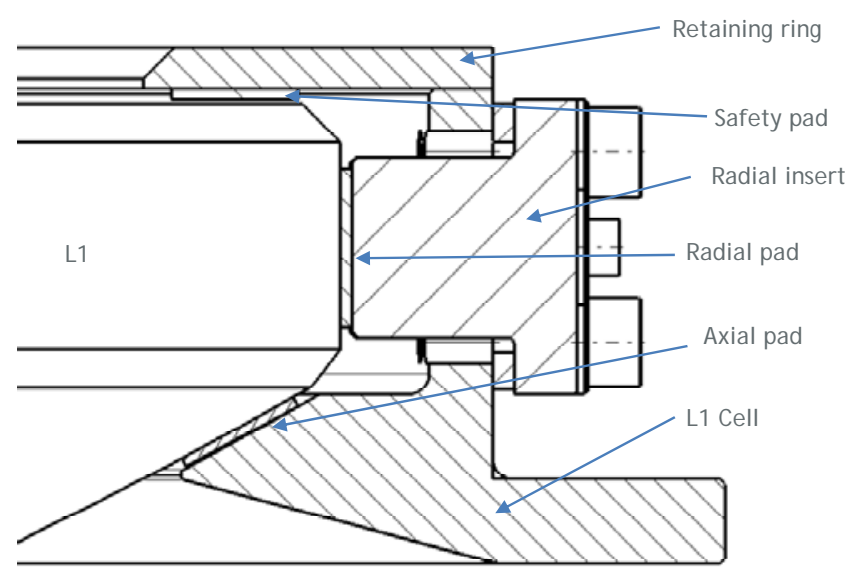

Figure 2. L1 and L1 Cell.

As shown in Table 1, the lenses are made in different materials. This imposes that their supports need to be also different to harmonize the different CTE's:

- Iron-nickel alloy with a very low CTE. Used for L1 and L6 cells and their corresponding extension rings to connect elastically to the rest of steel structure.

- Carbon steel (S355J2) for all the rests of cells and housings, cylindrical structures, etc.

Table 1. PFC Lens characteristics

\begin{tabular}{|l|c|c|l|}
\hline Lens & Diam.(mm) & Mass (kg) & Material \\
\hline L1 & 1100 & 230 & C7980 \\
\hline L2 & 661 & 94 & S-BSL7 \\
\hline L3 & 633 & 60 & PBL1Y \\
\hline L4 & 603 & 55 & PBL1Y \\
\hline L5 & 613 & 45 & NBK7 \\
\hline L6 & 587 & 39 & SK1300 \\
\hline
\end{tabular}

\subsection{RTV pads calculation and protoyping}

Axial pads are calculated to have deformations $<10 \%$ of the pad thickness as general criterial. Radial pads are dimensioned to have athermal design following the formulations included in the literature ${ }^{[3]}$. Results for the pads dimensions are included in the Table 2.

Table 2. Pads dimensioning

\begin{tabular}{|l|c|c|c|c|c|c|}
\hline & \multicolumn{3}{|c|}{ Axial Pad } & \multicolumn{3}{c|}{ Radial Pad } \\
\hline Lens & $\begin{array}{c}\text { Dimensions } \\
(\mathbf{m m})\end{array}$ & Shape factor & $\begin{array}{c}\text { E_RTV560 } \\
\text { corrected (MPa) }\end{array}$ & $\begin{array}{c}\text { Dimensions } \\
(\mathbf{m m})\end{array}$ & Shape Factor & $\begin{array}{c}\text { E_RTV560 } \\
\text { corrected (MPa) }\end{array}$ \\
\hline L1 & $15 \times 15 \times 1$ & 3.75 & 24 & $50 \times 50 \times 2.1$ & 5.9 & 128 \\
\hline L2 & $10 \times 20 \times 1$ & 3.33 & 20 & $40 \times 40 \times 2.9$ & 3.3 & 45 \\
\hline
\end{tabular}




\begin{tabular}{|l|c|c|c|c|c|c|}
\hline L3 & $10 \times 20 \times 1$ & 3.33 & 20 & $35 \times 35 \times 1.5$ & 5.8 & 124 \\
\hline L4 & $10 \times 20 \times 1$ & 3.33 & 20 & $35 \times 35 \times 1.5$ & 5.8 & 124 \\
\hline L5 & $10 \times 20 \times 1$ & 3.33 & 20 & $35 \times 35 \times 2.6$ & 2.9 & 36 \\
\hline L6 & $12 \times 12 \times 1$ & 3 & 17 & $15 \times 20 \times 1.1$ & 4.3 & 72 \\
\hline
\end{tabular}

The contact pressure for the axial pads shall be very uniform and equal between each of them for each lens. This implies that thickness tolerances need to be minimized and air bubbles need to be removed. To achieve that, several RTV560 pad samples were manufactured with different techniques. Best results (Figure 3) have been obtained by mixing RTV560 with a catalyst, placing it on a mould and curing it in a centrifuge machine.

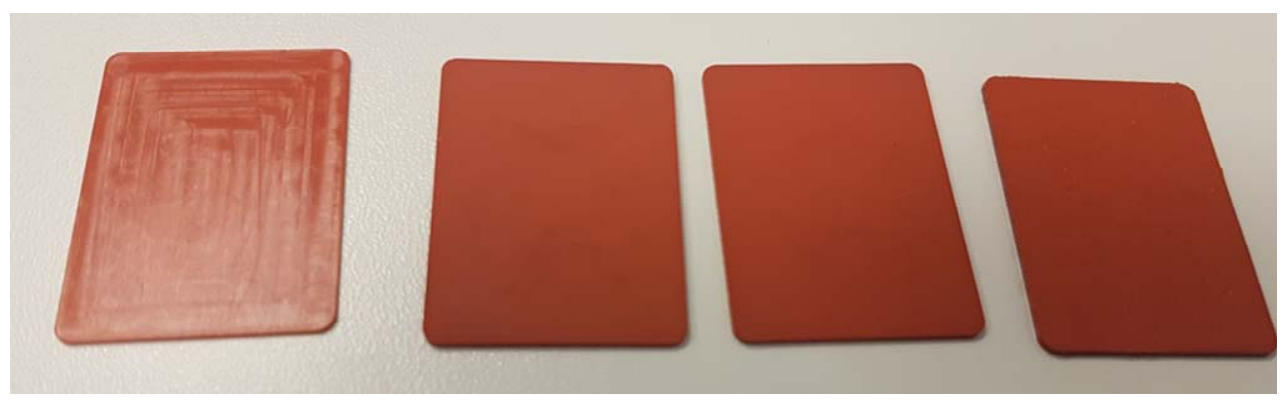

Figure 3. RTV pads prototype samples

\subsection{ADC's structure and driving system}

As mentioned, each ADC is composed of a cell doublet mounted in a driving mechanism. The cell assemblies of Lens 2 and Lens 3 are bolted together to form a common unit (ADC1) which is rotated in a bearing. The same applies to Lens 4 and Lens 5 for ADC2. Both ADC's are driven using a toothed crown fixed to Lens 3 Cell / Lens 5 Cell respectively and their corresponding anti-backlash pinion. The driving motor is fixed directly to a planetary gearbox with a gear ratio 1:100 to drive the anti-backlash pinion (Figure 4). 


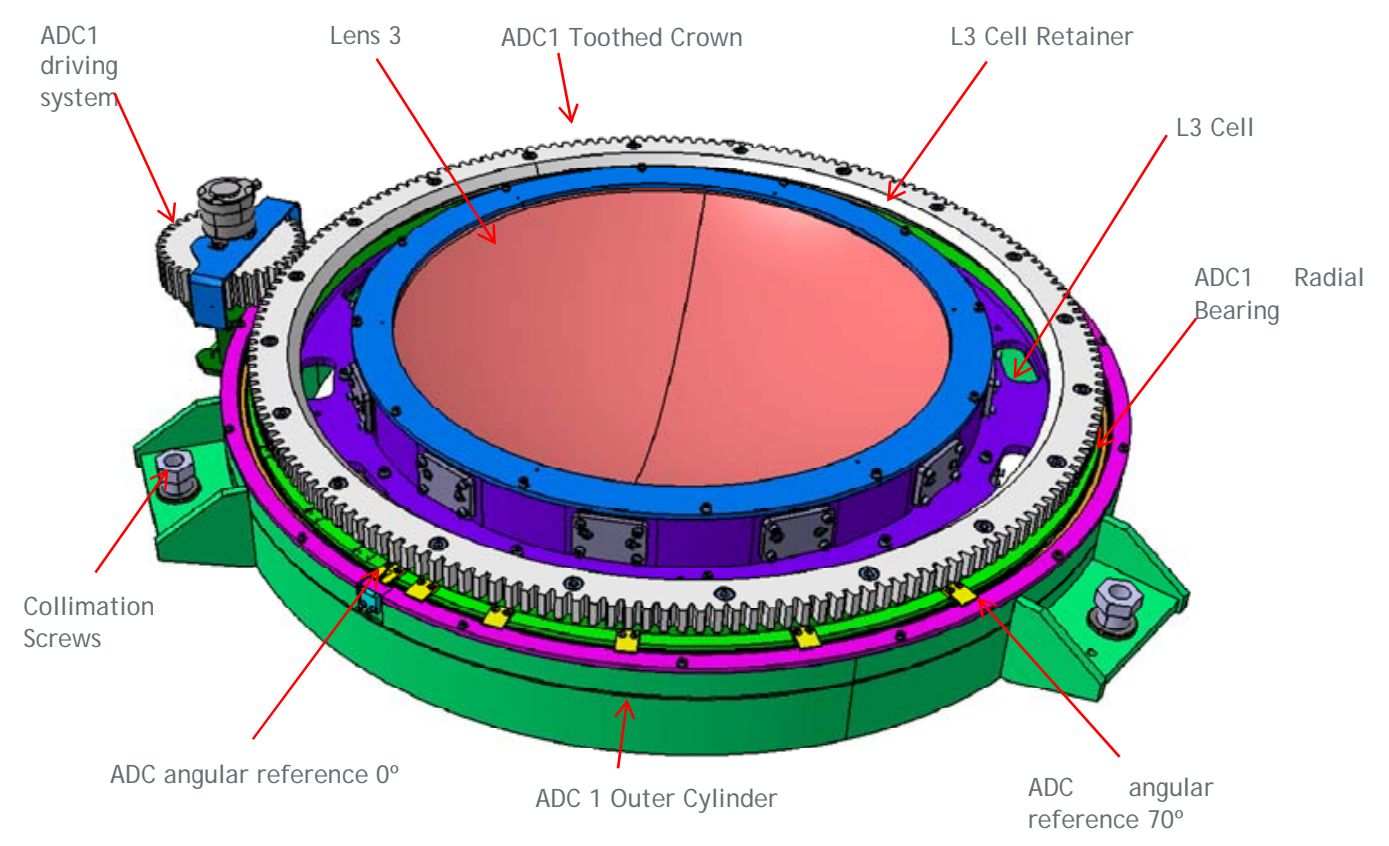

Figure 4.ADC structure

Gravitational displacement analysis performed on a FEM of the PFC confirms that the structure is stiff enough to fulfill the requirements for the operational loads. Obtained stress is also very low, showing safety margins above 10.
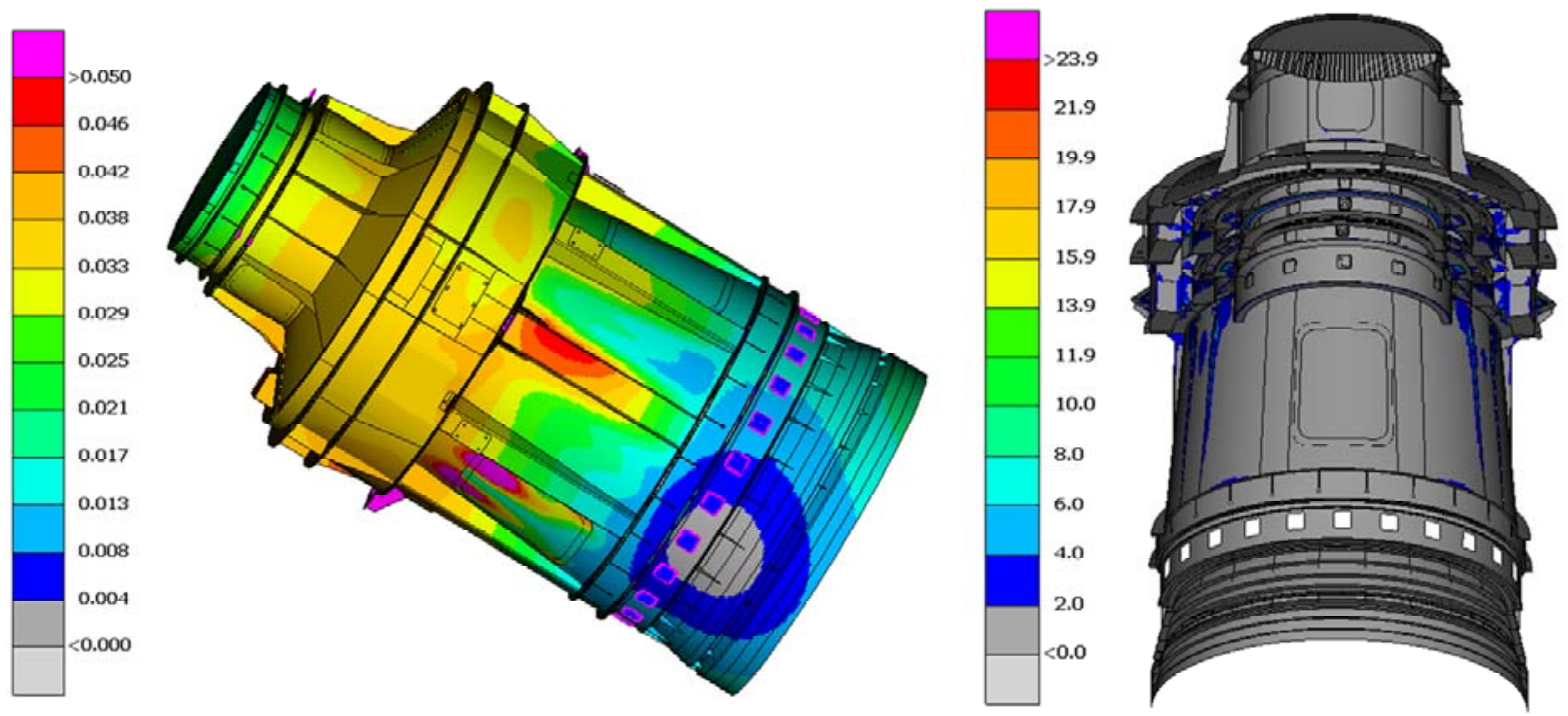

Figure 5. Left: Displacement (mm) when the PFC is $60^{\circ}$ from the vertical position with respect to L1. Right: Stress- Von Misses (MPa) in vertical position- 


\section{ASSEMBLY AND ALIGNMENT OF LENSES}

As done in other correctors like DeCam ${ }^{[4]}$ and DESI ${ }^{[5]}$, lenses of the WEAVE PFC will be first mounted and aligned into their respective lens cells and then into the PFC structural housing. To maintain the required image quality, the lenses need to be aligned to a high precision and maintain this position over a wide temperature range $\left(-5\right.$ to $\left.25^{\circ} \mathrm{C}\right)$ and different gravity vector. Alignment philosophy is as follows:

- Lens1-cell assembly together with the PFC main barrel assembly are the datum for assembly.

- The axis of rotation of the ADC bearings and the L1 axis are coaxially aligned.

- $\mathrm{ADC} 1$ followed by $\mathrm{ADC} 2$ are assembled into the bearings.

- Lens 6 optical axis is aligned with L1 optical axis.

Following this approach, alignment and positioning tolerances are given in Table 3.

Table 3. Alignment tolerances

\begin{tabular}{|c|c|c|c|c|}
\hline Datum axis & Measured axis & Axial ( $\mu \mathrm{m})$ & De-centring $(\mu \mathrm{m})$ & Tilt $\left(^{\circ}\right)$ \\
\hline L1 optical axis & $\begin{array}{l}\text { L1 assembly axis } \\
\text { (L1 optical axis) }\end{array}$ & NA & NA & NA \\
\hline L1 optical axis & $\begin{array}{c}\mathrm{ADC} 1 \text { axis of } \\
\text { rotation }\end{array}$ & \pm 50 & \pm 50 & $+/-0.008$ \\
\hline $\begin{array}{l}\mathrm{ADC} 1 \text { axis of } \\
\text { rotation }\end{array}$ & L2 assembly axis & \pm 75 & \pm 45 & $+/-0.008$ \\
\hline $\begin{array}{l}\text { ADC1 axis of } \\
\text { rotation }\end{array}$ & L3 assembly axis & \pm 75 & \pm 45 & $+/-0.008$ \\
\hline ADC1 Fixed part & $\begin{array}{l}\mathrm{ADC} 1 \text { axis of } \\
\text { rotation }\end{array}$ & \pm 25 & \pm 25 & $+/-0.002$ \\
\hline L1 optical axis & $\begin{array}{c}\mathrm{ADC} 2 \text { axis of } \\
\text { rotation }\end{array}$ & \pm 50 & \pm 50 & $+/-0.008$ \\
\hline $\begin{array}{l}\mathrm{ADC} 2 \text { axis of } \\
\text { rotation }\end{array}$ & L4 assembly axis & \pm 75 & \pm 45 & $+/-0.008$ \\
\hline $\begin{array}{c}\mathrm{ADC} 2 \text { axis of } \\
\text { rotation }\end{array}$ & L5 assembly axis & \pm 75 & \pm 45 & $+/-0.008$ \\
\hline L1 optical axis & $\begin{array}{l}\text { L6 assembly axis } \\
\text { (L6 optical axis) }\end{array}$ & \pm 50 & \pm 50 & $+/-0.010$ \\
\hline
\end{tabular}

Allowed gravity displacement are included in the table below.

Table 4. Displacement tolerances

\begin{tabular}{|c|c|c|c|c|}
\hline Datum axis & Measured axis & $\begin{array}{c}\text { Axial } \\
(\boldsymbol{\mu m})\end{array}$ & De-centring $(\boldsymbol{\mu m})$ & Tilt $\left.\mathbf{(}^{\mathbf{0}}\right)$ \\
\hline L1 optical axis & $\begin{array}{c}\text { L1 assembly axis } \\
\text { (L1 optical axis) }\end{array}$ & NA & NA & NA \\
\hline L1 optical axis & $\begin{array}{c}\text { ADC1 axis of } \\
\text { rotation }\end{array}$ & \pm 25 & \pm 40 & $+/-0.008$ \\
\hline L1 optical axis & $\begin{array}{c}\text { ADC2 axis of } \\
\text { rotation }\end{array}$ & \pm 25 & \pm 40 & $+/-0.008$ \\
\hline L1 optical axis & $\begin{array}{c}\text { L6 assembly axis } \\
\text { (L6 optical axis) }\end{array}$ & \pm 20 & \pm 30 & \pm 0.01 \\
\hline
\end{tabular}

Verification of alignment is done through mechanical tools (dial length gauges, caliper tools) attached to precision alignment tables (Figure 6). Verification of the complete PFC alignment will be performed by means of a laser tracker. 


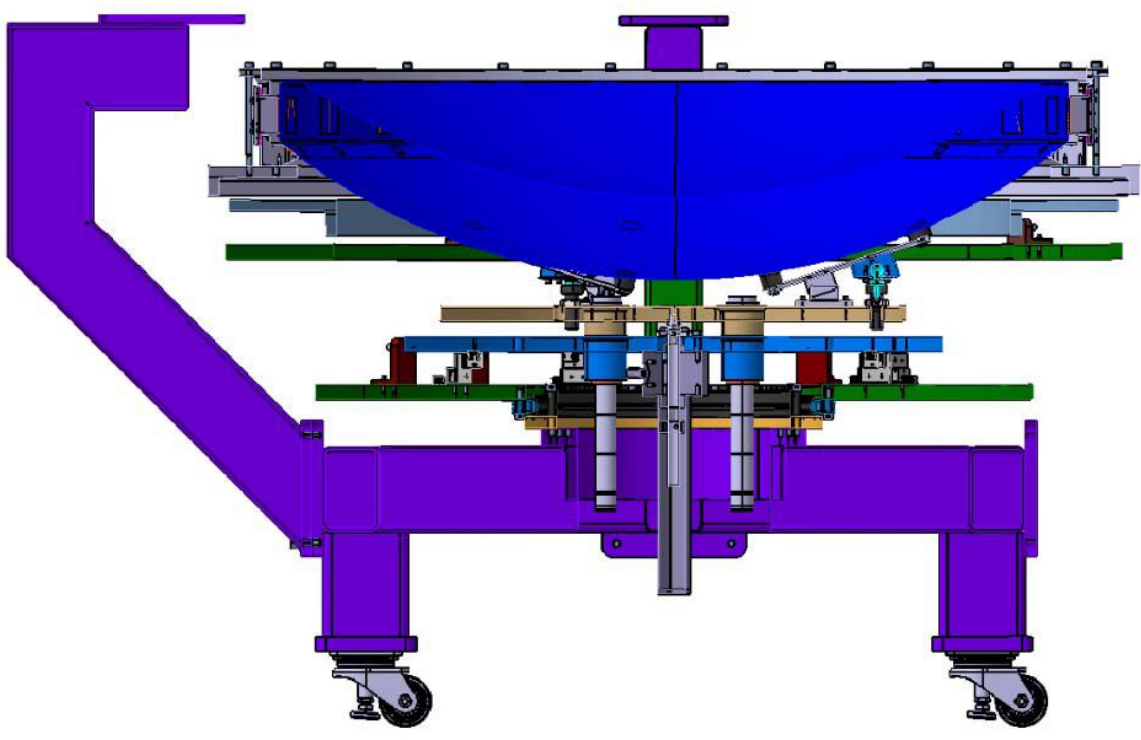

Figure 6. Lateral view of alignment table

Alignment tables are based on a rotary table having very low axial and radial runout ( 3 to $6 \mu \mathrm{m}$ ) and XY plus collimation adjusting bolts for cell and lens separately. Each cell is placed in the alignment table and centered with respect to the rotation axis using dial gauges and XY adjusting bolts. Tilt is adjusted using the collimation screws. The lens is placed inside its cell first via a screw jacket. Fine adjustment is performed via the collimation screw.

In order to validate the assembly and alignment process, before mounting the lenses the PFC will be assembled using dummy lenses. Dummies have been designed with same mass and center of gravity than lenses and similar shape This will allow gaining experience with lenses manipulation and also to measure the gravitational displacement of the lenses caused by structures deflection. Axial and tilt displacements of each dummy lens for the different gravity orientations will be measured using carbon fibre-reinforced polymer (CFRP) bars and linear gauges (Figure 7). Lateral displacement will be measured using a collimated laser light source placed at the central hole on dummy Lens 1 and a quadrant detector is used to cover the aperture from the hole on dummy Lens 6. 


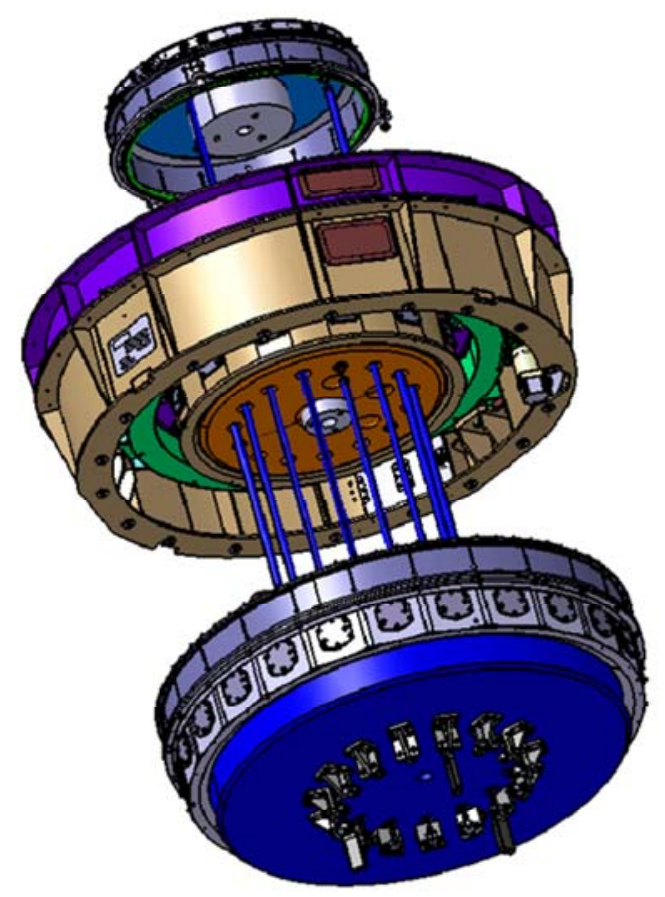

Figure 7. Lateral view of alignment table

\section{CURRENT STATUS}

Detailed design activities of the Prime Focus Corrector have been completed. Performed analysis show that the design is compliant with the required specifications. An assembly and alignment procedure has been defined to meet the strict tolerance values. Manufacturing of mechanical pieces is currently ongoing. Integration and alignment activities are planned to be performed during 2018.

\section{ACKNOWLEDGEMENTS}

WEAVE PFC has been designed by SENER contracted by IAC with the technical support of ING.

We would like to thank all the people involved in the project for their inputs to the PFC design and the definition of the alignment technique. Especially, thanks to the ING and IAC teams for their contributions during the project reviews and the management performed during the currently running project phases. 


\section{REFERENCES}

[1] Canchado, M., Romero A., Maroto, O., Tomàs, A., et al. "The WEAVE focus translation system: from design to construction", Proc. SPIE 9912, 9912 - 9912 - 15 (2016).

[2] Dalton, G.; Trager, S.; Abrams, D.C.; Bonifacio, P., et al., "Final design and progress of WEAVE: the next generation wide-field spectroscopy facility for the William Herschel Telescope", Proc. SPIE 9908, 9908 - 9908 -10 (2016).

[3] Doyle, k., Michels G., Genberg, V., “Athermal design of nearly incompressible bond”, Proc. SPIE 4471, 4771 $4771-8$ (2002).

[4] Doel, P., Brooks, D., Antonik M.L., Flaugher, B., et al., "Assembly, alignment, and testing of the DECam wide field corrector optics", Proc. SPIE 8446, 8446 - 8446 - 9 (2012).

[5] Brooks, D.; Doel, P., Besuner, R. Flaugher, B.; et al., "The alignment and assembly of the DESI prime focus corrector", Proc. SPIE 9908, 9908 - 9908 - 8 (2016). 